\title{
Thyrotoxicosis Leading to Adrenal Crises Reveals Primary Bilateral Adrenal Lymphoma - Case Report
}

C. Roque, R. Fonseca, C. Bello, C. Moniz, R.Ferreira, C. Vasconcelos

Endocrinology Department - Hospital de Egas Moniz, Centro Hospitalar de Lisboa Ocidental, E.P.E.

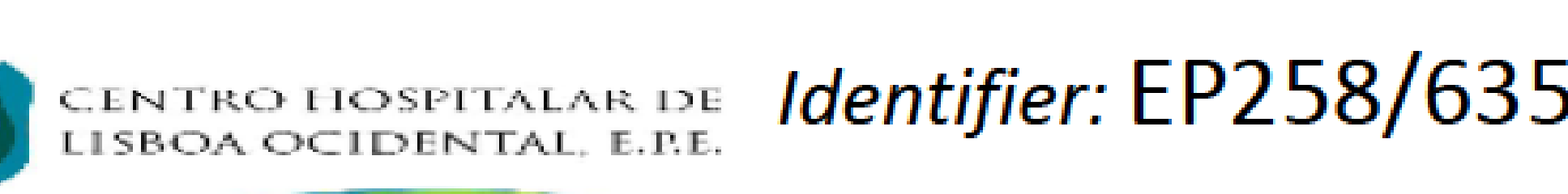
Lisbon, Portugal

\section{Introduction}

Amiodarone use may be associated with secondary severe organ dysfuntion. Thyrotoxicosis develops in $15 \%$ cases. Primary bilateral adrenal lymphoma is a rare malignancy. It frequently presents bilaterally and with symptoms of adrenal insufficiency. Symptomatology for both conditions is nospecific, especially in the elderly population, and a high suspition index is necessary to reach appropriate diagnosis.

\section{Case Report}

Female gender, 78 year old. Institutionalized. Personal history: Hypertension, Atrial Fibrillation and Diabetes Mellitus for 15 years without complications. Medication: perindopril, amiodarone, sinvastatin, metformin, trazodone. She had recently been prescribed antibiotic for UTI complicated by vomiting and hypochloremic hyponatremia. Trazodone was stoped.
Referred to the Emergency Department due to confusion, nausea and vomiting.

She said she did not complied with the prescribed antibiotic for the UTI. At physical examination: diffuse abdominal pain on palpation, slight dehydration. Laboratory: Leucocyturia without leucocytosis, PCR 7,4. Na $125 \mathrm{mmol} / \mathrm{l}, \mathrm{K}$ 4,56 $\mathrm{mmol} / \mathrm{l}, \mathrm{TSH} 0,01 \mathrm{uU} / \mathrm{ml}, \mathrm{FT} 468$ (10-18) pmol/l, FT3 6,34 (4-8) pmol/l, negative anti-TPO and anti-TGL.

Admitted diagnosis: Urinary tract infection and thyreotoxicosis.
Transfered to the Endocrinology ward.

Thyroid US

Heterogenous multinodular goiter.

Doppler evatuation showed decreased vascular signal.

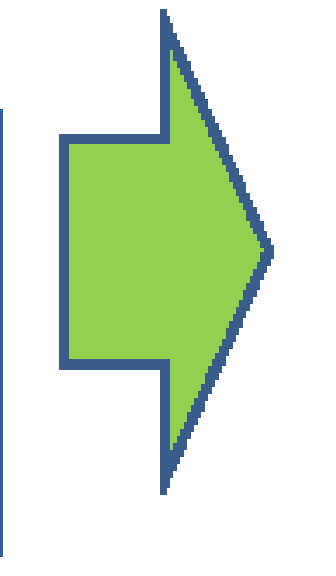

Type 2 Amiodarone Induced Thyroiditis
Treatment: Prednisolone $40 \mathrm{mg} / \mathrm{d}$, Tiamazol $30 \mathrm{mg} / \mathrm{d}$. Targeted antibiotic. Maintainance fluids.

Clinical deterioration

\section{Only sepsis? Adrenal Insufficiency?}

Fluid ressuscitation and hydrocortisone led to amelioration of the hemodynamic instability and clinical improvement, with high dose hydrocortisone requirements for stability.
Nosocomial pneumonia

Fever

Worsening of thyroid funcion

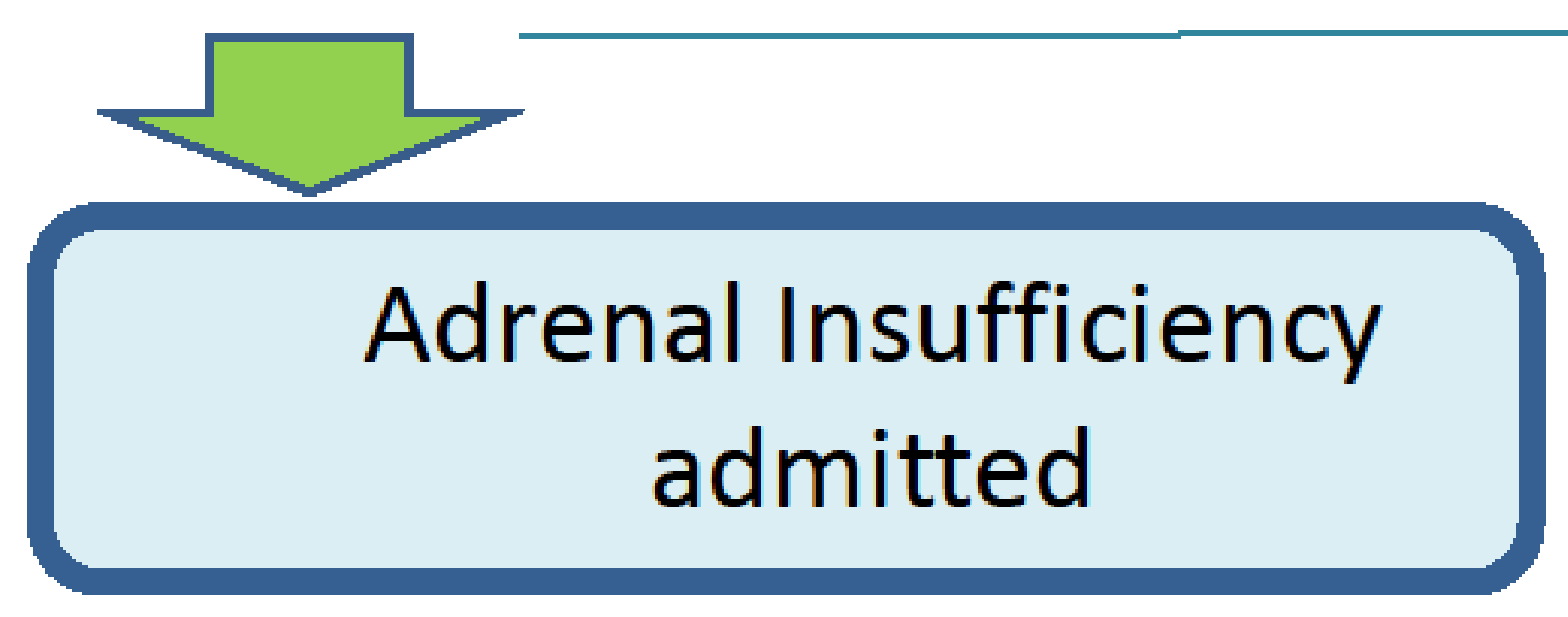

No clinical contitions to switch/stop hydrocortisone and perform short synacthen.

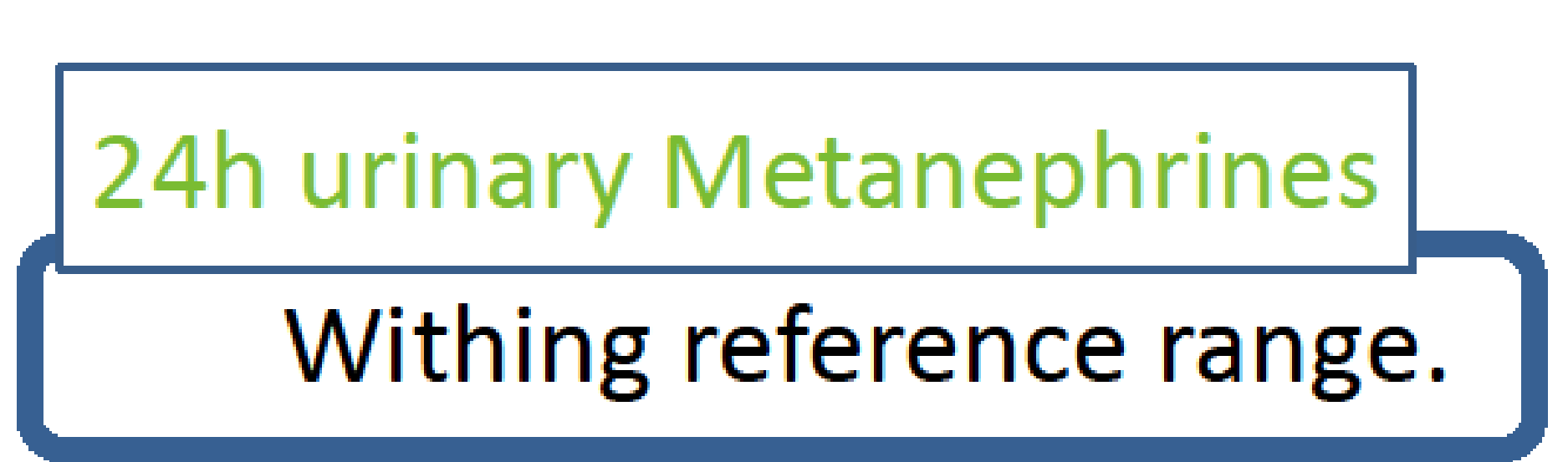

$$
\mathrm{B}
$$$$
\text { Bronchofibroscopy }
$$

Endobronchic primary lesion ? No malignant cells in the BAL/brushing.

\section{Abdominal US}

Right and left justa-renal heterogenous solid nodules $(6,6$ and $7 \mathrm{~cm}$ respectively) and pleural effusion.

Hyponatremia, hypoglycemia and vomiting

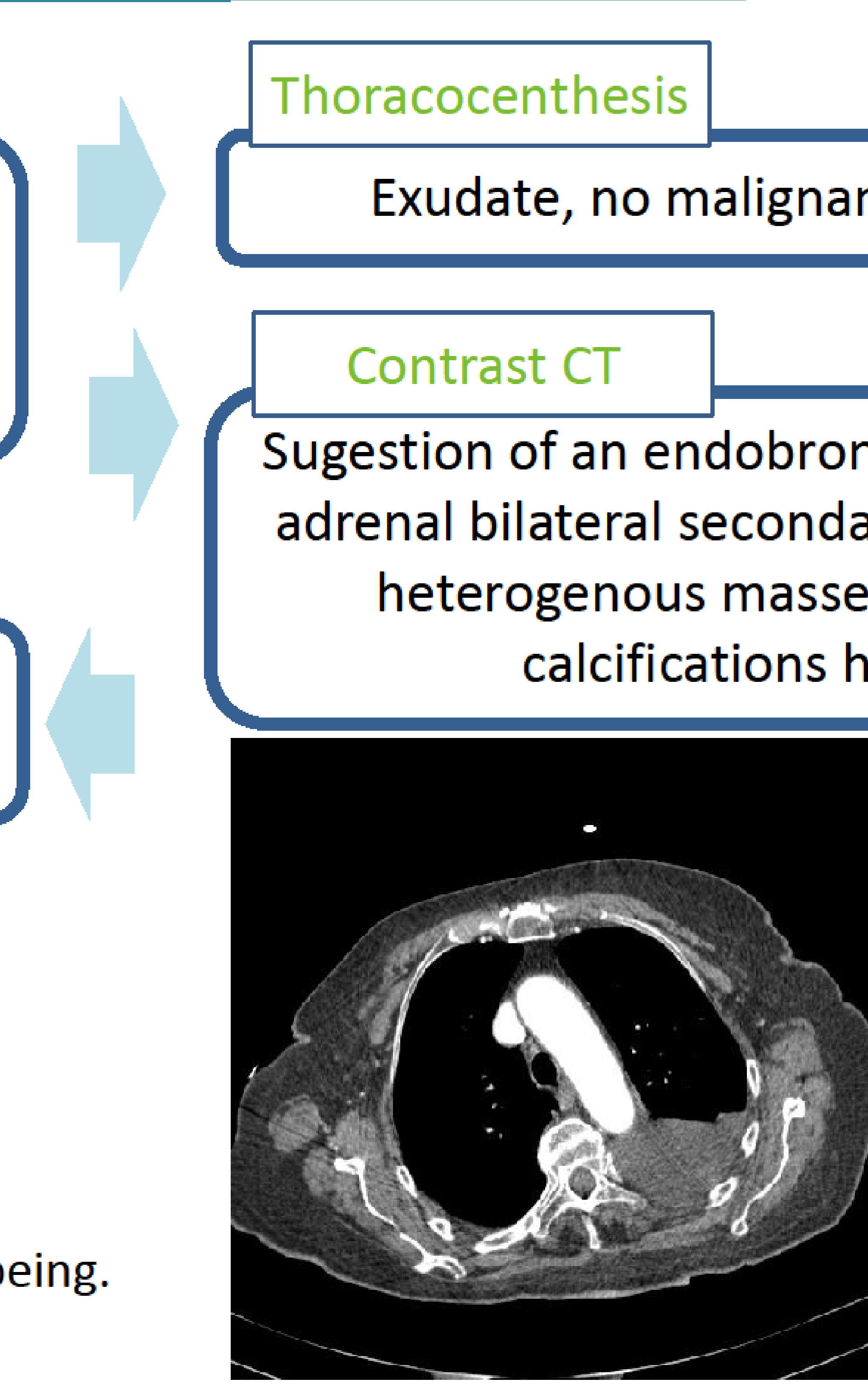

Left pleural effution.

Durig the investigation the patient had a favorable couse.

She was clinically stable, parcially oriented, minimally cooperative and reported well being. After this period of seemingly favourable evolution:

\section{Cardiorrespiratory arrest.} no patient colaboration.

\section{Conclusion}

Primary adrenal lymphoma is a rare cause of adrenal insufficiency. Although rare it must allways integrate the differential diagnosis, specially in the elderly patient where symptoms are subtle and progression is often fast and fatal. Thyrotoxicosis, specially when amiodarone-induced, may be difficult to control rapidlly. Transient periods of worsening thyroid funtion acompain infectious processes. Thyreotoxicosis worsens the adrenal insufficiency picture leading to increased need of substitutive dose requirements and dose ajustments. Mortality rises significantly. 